\title{
TWO THEOREMS ON EXCELLENT RINGS ${ }^{1)}$
}

\author{
SILVIO GRECO
}

Let $f: A \rightarrow B$ be a homomorphism of commutative noetherian rings. The main results of this paper are:

(a) Assume $f$ is finite and induces a surjective map on the spectra. Then if $B$ is quasi-excellent $A$ is quasi-excellent and is excellent if it is universally catenarian (Th. 3.1$)^{2)}$; and

(b) If $f$ is absolutely flat and $A$ is excellent then $B$ is excellent (Th. 5.3). In particular the strict henselization of an excellent local ring is excellent (Cor. 5.6.).

To prove the above we give some more general partial results. For instance in section 1 we study the finite descent for certain $P$-homomorphisms and $P$-rings, and in section 2 we show that several properties related with the openness of loci (such as property J2 of excellent rings) descend by surjective scheme morphisms locally of finite type. Result (a) is given in section 3 together with some applications, while in section 4 we show with a counterexample that the assumption " $f$ finite" cannot be replaced by " $f$ of finite type". Section 5 contains result (b), which generalizes the main statements of [5]. This is possible by a theorem of André [1] and a new proof for the chain condition.

The author wishes to thank Prof. H. Matsumura for encouraging him to write this paper and for several useful conversations on its content.

Foreword. All rings are assumed to be commutative and noetherian. We use freely the notations and the definitions of [9] and [7].

\section{Finite descent for $\boldsymbol{P}$-homomorphisms and $\boldsymbol{P}$-rings.}

Let $A$ be an algebra over the field $k$ and let $P$ be any of the fol-

Received December 25, 1974.

Revised June 4, 1975.

1) This research was supported by CNR.

2) This result was announced at the CNR-GNSAGA meeting held in Siena (Italy) in the fall 1973. 
lowing properties: geometrically regular (or normal or reduced); CohenMacaulay; $S_{n} ; R_{n}$; etc ; (see [8], 7.3).

1.1. Definition. A ring homomorphism is a P-homomorphism if it is flat and its fibers have property $P$. A ring is a $P$-ring if its formal fibers at the maximal (or prime) ideals have $P$ (l. cit.).

1.2. Proposition. Let $f: A \rightarrow B$ be a flat ring homomorphism and let $A^{\prime}$ be a finite A-algebra such that $\operatorname{Spec}\left(A^{\prime}\right) \rightarrow \operatorname{Spec}(A)$ is onto. Then $f$ is a P-homomorphism if and only if the induced map $f^{\prime}: A^{\prime} \rightarrow B^{\prime}=$ $B \otimes_{A} A^{\prime}$ is a P-homomorphism.

Proof. The "only if" is known (l. cit.). Conversely let $\mathfrak{p} \in \operatorname{Spec}(A)$ and let $\mathfrak{p}^{\prime}$ be a prime ideal of $A^{\prime}$ lying over $\mathfrak{p}$. We have $\left(B \otimes_{A} k(\mathfrak{p})\right)$ $\otimes_{k(\mathfrak{p})} k\left(\mathfrak{p}^{\prime}\right)=B^{\prime} \otimes_{A^{\prime}} k\left(\mathfrak{p}^{\prime}\right)$ and since $k\left(\mathfrak{p}^{\prime}\right)$ is finite over $k(\mathfrak{p})$ the conclusion follows by [8], 6.7.8.

1.3. Proposition. Let $A, A^{\prime}$ be as in 1.2. Then the following are equivalent:

i) $A$ is a P-ring;

ii) $A^{\prime}$ is a P-ring;

iii) Any faithfully flat $f: A \rightarrow B$ whose closed fibers (i.e. the fibers at the maximal ideals) are geometrically regular is a P-homomorphism.

Proof. By [8],7.3. i) implies ii). Conversely let $\mathfrak{m}$ be a maximal ideal of $A$. Then $C=A^{\prime} \otimes_{A} A_{\mathrm{m}}$ is a semilocal $P$-ring and the canonical map $\operatorname{Spec}(C) \rightarrow \operatorname{Spec}\left(A_{\mathrm{m}}\right)$ is onto. Moreover if $\hat{C}$ is the completion of $C$, the canonical map $C \rightarrow \hat{C}$ is a $P$-homomorphism ([8], 7.4.6), and i) follows by 1.2. Now we prove that i) implies iii). We may assume that $f$ is a local homomorphism of local rings. Let $\hat{f}$ be the extension of $f$ to the completions, and let $u, v$ be the embeddings of $A, B$ in their completions. Then $\hat{f}$ is formally smooth (since $f$ is, see [8], 0.19.7.1), and hence is regular by [1]. Then $v \circ f=\hat{f} \circ u$ is a $P$-homomorphism and $u$ is a $P$-homomorphism by faithful flatness ([8],7.3). Thus $f$ is a $P$ homomorphism. To prove that iii) implies i) one can apply iii) to the canonical maps $A \rightarrow A \times\left(A_{\mathrm{m}}\right)^{\wedge}$ (direct product of rings), where $\mathfrak{m}$ is any maximal ideal.

1.4. COROLlary. Let $A \rightarrow B$ be an injective homomorphism of finite type and assume $B$ is a domain and a P-ring. Then there is a non zero 
$f \in A$ such that $A_{f}$ is a P-ring.

Proof. By the normalization lemma it follows easily that there is a nonzero $f \in A$ such that $B_{f}$ is a finite overring of $C=A_{f}\left[X_{1}, \cdots, X_{n}\right]$ where the $X_{i}$ 's are indeterminates (see e.g. [2], p. 70, ex. 20). The conclusion follows easily by $\mathbf{1 . 3}$.

1.5. Remarks. i) In [13] (while studying the Picard group of certain ring extensions) it is shown how to obtain a ring $A$ by "glueing" prime ideals of a ring $A^{\prime}$, and conditions are given for $A^{\prime}$ to be finite over $A$. Hence the above results can be applied.

ii) $A$ local ring is Nagata if and only if any formally smooth local homomorphism $A \rightarrow B$ is reduced. This follows by 1.3 and [8],7.4.6 and 7.7.2.

iii) If $A$ is a $P$-ring and $A \rightarrow B$ is a reduced homomorphism then $A_{\mathfrak{p}} \rightarrow B_{\mathfrak{p}}$ is a $P$-homomorphism whenever $\mathfrak{B}$ is a minimal prime of $\mathfrak{p} B$. This follows by 1.3 .

iv) If $A$ is a quotient of a Cohen-Macaulay local ring any formally smooth local homomorphism $A \rightarrow B$ is Cohen-Macaulay, as follows by 1.3 and [6], 13.6 .

v) The implication ii) $\Rightarrow$ i) of 1.3 is false if $A^{\prime}$ is only of finite type over $A$ : see 4.1 below.

\section{Descent of openness of loci and of property $\mathbf{J 2}$.}

If $P$ is a property of local rings and $X$ is a scheme (locally noetherian as always) we denote by $U_{P}(X)$ the set of points $x \in X$ such that the local ring $O_{X, x}$ has property $P$. If $X=\operatorname{Spec}(A)$ we write also $U_{P}(A)$.

2.1. Proposition. Let $X$ be an irreducible scheme, $Y$ an integral scheme and $f: X \rightarrow Y$ a dominant morphism locally of finite type. Let $P$ be any property of local rings which descends by faithful flatness. Then if $U_{P}(X)$ contains a nonempty open set the same is true for $U_{P}(Y)$.

Proof. Since any two nonempty open sets in an irreducible scheme have a nonempty intersection we may assume that ( $X$ and $Y$ are affine and) $f$ is of finite type. By the theorem of generic flatness ([8],6.8.1) there is a nonempty open set $U \subset Y$ such that the restriction $g$ of $f$ to $f^{-1}(U)$ is flat. Since $g$ is clearly of finite type it is also open ([8], 2.4.6) and the conclusion follows easily by our assumptions on $X$ and $Y$. 
2.2. COROLlary. Let $A \rightarrow B$ be a finite type injective homomorphism of integral domains. Then if $U_{P}(B)$ ( $P$ as in 2.1) contains a nonempty open set the same is true for $U_{P}(A)$.

A scheme is said to be $\mathrm{J} 2$ if it has an open affine covering whose rings are J2. Then any open subscheme is $\mathrm{J} 2$.

2.3. Proposition. Let $f: X \rightarrow Y$ be a surjective scheme morphism locally of finite type. Then $X$ is $J 2$ if and only if $Y$ is J2.

Proof. The "if" part is clear by definition (see [9]). To prove the converse we may assume that $Y$ is affine and $f$ is of finite type. Then $f$ is quasi-compact, whence $X=V_{1} \cup \cdots \cup V_{n}$ where the $V_{i}$ 's are affine and open. Let $Z$ be the disjoint union of the $V_{i}$ 's. Then $Z$ is affine and J2, and $f$ induces a surjective morphism of finite type $g: Z \rightarrow Y$. Thus it is enough to prove:

2.4. COROLlaRY. Let $\varphi: A \rightarrow B$ be a finite type ring homomorphism which induces a surjective map on the spectra. Then $B$ is $J 2$ if and only if $A$ is J2.

Proof. We have to show that, for any finite integral $A$-algebra $A^{\prime}, \operatorname{Reg}\left(A^{\prime}\right)$ contains a nonempty open set. By assumption the ring $B^{\prime \prime}=A^{\prime} \otimes_{A} B$ is not zero. Let $\mathfrak{p}$ be a minimal prime of $B^{\prime \prime}$ and let $B^{\prime}=B^{\prime \prime} / \mathfrak{p}$. Then $\operatorname{Reg}\left(B^{\prime}\right)$ is open and nonempty and the conclusion follows by 2.2 .

A scheme is said to be a Nagata scheme if it has an open affine covering whose rings are Nagata. Then any open subscheme is Nagata.

2.5. Proposition. Let $f: X \rightarrow Y$ be as in 2.3. Then the following are equivalent:

i) $Y$ is Nagata;

ii) $X$ is Nagata and $Y$ is locally Nagata (i.e. $O_{Y, y}$ is Nagata for any $y \in Y$ ).

Proof. It is clear that i) implies ii). To prove the converse recall that a noetherian ring $A$ is Nagata if and only if it is locally Nagata and Nor $(B)$ contains a nonempty open set for any finite integral $A$ algebra $B$ ([8], 7.7.2). The conclusion follows as in 2.3 (with "normal" in place of "regular"). 
2.6. Remarks. i) The implication ii) $\Rightarrow$ i) in 2.5 is false if $Y$ is not locally Nagata: see 4.1 .

ii) We do not know if 2.3 and 2.5 hold if $f$ is only dominant.

\section{Finite descent for excellent rings.}

A ring $A$ is said to be quasi-excellent $(Q E)$ if it is a $G$-ring and is J2. Then $A$ is excellent if it is universally catenarian (UC). By 1.3 (with $P=$ geometrically regular) and 2.4 we have:

3.1. THEOREM. Let $A \rightarrow A^{\prime}$ be a finite ring homomorphism which induces a surjective map on the spectra. Then:

i) $A$ is $Q E$ if and only if $A^{\prime}$ is $Q E$;

ii) $A$ is excellent if and only if it is $U C$ and $A^{\prime}$ is $Q E$.

3.2. CoRollary. Let $A \rightarrow A^{\prime}$ be a finite injective homomorphism of integral domains. Assume either i) $f$ is flat; or ii) $A$ is normal. Then $A$ is excellent if and only if $A^{\prime}$ is excellent.

Proof. By 1.3 we have to show that $A$ is UC if $A^{\prime}$ is. Since i) and ii) are inherited by polynomials and fractions, it is sufficient to show that $A$ is catenarian, and we may assume $A$ is local. The conclusion follows then by [9], sec. 5 and

3.3. Lemma. Let $A, A^{\prime}$ be two domains with $A$ local and let $f: A \rightarrow A^{\prime}$ be a homomorphism such that: i) the going down theorem holds, and ii) $\operatorname{dim} A / \mathfrak{p}=\operatorname{dim} A^{\prime} / \mathfrak{p} A^{\prime}$ for all $\mathfrak{p} \in \operatorname{Spec}(A)$. Then if $A^{\prime}$ is catenarian the same is true for $A$.

Proof. By Ratliff [14] a local domain $C$ is catenarian if and only if $\operatorname{dim} C_{\mathfrak{p}}+\operatorname{dim} C / \mathfrak{p}=\operatorname{dim} C$ for all $\mathfrak{p} \in \operatorname{Spec}(C)$. Let then $\mathfrak{p}$ be a prime ideal of $A$ and let $\mathfrak{p}^{\prime}$ be a minimal prime of $\mathfrak{p} A^{\prime}$ such that $\operatorname{dim} A^{\prime} / \mathfrak{p}^{\prime}=$ $\operatorname{dim} A / \mathfrak{p} . \quad$ It is easy to see that $f^{-1}\left(\mathfrak{p}^{\prime}\right)=\mathfrak{p}$, whence $\operatorname{dim} A_{\mathfrak{p}}=\operatorname{dim} A_{\mathfrak{p}^{\prime}}^{\prime}$ ([9], section 5). It is also clear that $\operatorname{dim} A=\operatorname{dim} A_{\mathfrak{m}^{\prime}}^{\prime}$ for all maximal ideals $\mathrm{m}^{\prime}$ of $A^{\prime}$. The conclusion follows easily.

3.4. Corollary. Let $f: A \rightarrow A^{\prime}$ be an injective ring homomorphism of finite type. Assume $A^{\prime}$ is a $Q E$ domain (resp. $A^{\prime}$ is an excellent domain and $A$ is normal). Then there is a nonzero $f$ in $A$ such that $A_{f}$ is $Q E$ (resp. excellent).

Proof. It follows by 3.1 and 3.2 with the same argument used in 
the proof of 1.4 .

3.5. CoRollary. Let $A$ be a ring and let $A^{\prime}$ be the integral closure of $A_{\text {red }}$ in its total ring of fractions. Then $A$ is $Q E$ if and only if $A^{\prime}$ is $Q E$ and is finite over $A$.

Proof. It is an easy consequence of 1.3 .

3.6. Corollary. Let $A$ be a one-dimensional excellent ring. Assume that $A / \mathfrak{p}$ has characteristic zero for any minimal prime $\mathfrak{p}$, and that the fields $A / \mathfrak{m}$ are perfect for any maximal ideal $\mathfrak{m}$ of $A$. Then the ring $B=A\left[X \ldots X_{n}\right]\left[\left[Y, \ldots, Y_{m}\right]\right]$ is excellent for all $m, n$.

Proof. $B$ is UC by [15], and the excellence of $B$ was proved by Nomura [11] when $A$ is a Dedekind domain. Since our assumptions on $A$ are easily seen to hold for the integral closure of $A_{\text {red }}$, the conclusion is an easy consequence of 3.5 .

3.7. Proposition. Let $A$ be a ring of dimension $\leqslant 2$, and assume $A / \mathfrak{P}$ has characteristic zero for any minimal prime $\mathfrak{R}$. Let a be an ideal of $A$ and let $B$ be the a-completion of $A$. Then if $A$ is excellent the same is true for $B$.

Proof. $B$ is UC by [15], and thus it is sufficient to show that $B$ is QE. Since $A$ is a $G$-ring, the canonical homomorphism $A \rightarrow B$ is regular ([8],7.8.3 v)), and hence the integral closure $B^{\prime}$ of $B_{\text {red }}$ is canonically isomorphic to $B \otimes_{A} A^{\prime}$ where $A^{\prime}$ is the integral closure of $A_{\text {red }}$ ([8],6.14). Then by 3.5 we may assume that $A$ is a normal domain, so that $B$ is a normal ring (l. cit.).

Let $\mathfrak{M}$ be a maximal ideal of $B$ and $\mathfrak{m}$ its contraction to $A$. Since the local rings $A_{\mathrm{m}}$ and $B_{\mathfrak{m}}$ have the same completion $([6], 6.2)$ and $A_{\mathfrak{m}}$ is a $G$-ring, it follows that the generic formal fiber of $B_{\mathfrak{m}}$ is regular and hence geometrically regular by our assumption on the characteristic. Moreover if $\mathfrak{B} B_{\mathfrak{m}}$ is a nonzero prime ideal, the ring $B / \mathfrak{P}$ is either complete semilocal (if $\mathfrak{\beta} \not \subset \mathfrak{a} B$ ) or is a quotient ring of $A$. In both cases it is excellent and then formal fiber of $B_{\mathfrak{m}}$ at $\mathfrak{\beta} B_{\mathfrak{m}}$ is geometrically regular. This proves that $B$ is a $G$-ring.

Since $A \rightarrow B$ is regular, $\operatorname{Reg}(B)$ is the preimage of $\operatorname{Reg}(A)$ by the canonical map $\operatorname{Spec}(B) \rightarrow \operatorname{Spec}(A)$, and hence it is open. But $B$ is normal and then it follows that $\operatorname{Reg}(B / \mathfrak{B})$ is open and nonempty for any 
minimal prime $\mathfrak{\beta}$ of $B$. Finally by the previous argument and the assumption on the characteristic it follows that $B$ is J2. This completes the proof.

3.8. Remarks. i) As we have seen in 1.5 i), theorem 3.1 can be applied to rings obtained by glueing prime ideals.

ii) It is false in general that if $A \rightarrow A^{\prime}$ is finite and injective and $A^{\prime}$ is UC then $A$ is $\mathrm{UC}$ ([8], 5.6.11).

iii) The hypothesis "finite" in 3.1 cannot be replaced by "of finite type", see 4.1 below. However the following conjecture seems reasonable: Let $f: X \rightarrow Y$ be a proper surjective morphism of locally noetherian schemes. Then if $X$ is QE, $Y$ is QE. In the affine case this is just 3.1. Note also that by 3.1 and the Stein factorization one may assume $f_{*} O_{X}=O_{Y}$.

iv) It is not known if an ideal-adic completion of an excellent ring is excellent. Interesting contributions to this problem can be found in [10], [11], [17], [19], [20].

\section{A counterexample.}

We show that some hypotheses in some of the previous results cannot be weakened (see: $1.5 \mathrm{v}$ ) ; 2.6 i) ; 3.8 iii)).

4.1. Proposition. There are two local domains of dimension $1 \mathrm{~A}$, $B$ and a ring homomorphism $f: A \rightarrow B$ such that:

i) $f$ is local, of finite type and birational (i.e. it induces an isomorphism of the fields of fractions);

ii) $B$ is an excellent $D V R$ and $A$ is non-Nagata.

To prove the above we need the following generalization due to Valabrega of a well-known construction of Nagata.

4.2. Proposition. Let $X, Y$ be analytically independent over the field $k$ and let $f \in k[X][[Y]]$ be transcendental over $k(X, Y)$. Let $C=$ $k(X, Y, f) \cap k[[X, Y]]$. Then:

i) $C$ is a 2-dimensional regular local ring, $X, Y$ is a regular system of parameters of $C$ and $\hat{C}=k[[X, Y]]$;

ii) $A=C / f C$ is a domain and $\hat{A}=k[[X, Y]] /(f)$.

Proof. See [18], Prop. 3 and proof of Prop. 7. 
Proof of 4.1. Notations are as in 4.2. Let $g, h \in k[X][[Y]]$ be algebraically independent over $k(X, Y)$ and put $f=g h^{2}$. Then $f$ is transcendental over $k(X, Y)$; moreover, after changing a finite number of terms we may assume that the leading term of $f$ is $X Y^{2}$. Let $A=C / f C$, where $C=k(X, Y, f) \cap k[[X, Y]]$. By $4.2 A$ is a local domain of dimension one, and $\hat{A}=k[[X, Y]] /(f)$; hence $A$ is not a Nagata ring.

Now we construct $B$. Let $\mathfrak{m}$ be the maximal ideal of $A$ and let $Z$ be the blow-up of $\operatorname{Spec}(A)$ along $m$. Let $E$ be the exceptional divisor (i.e. the closed subscheme of $Z$ associated to the sheaf $\mathrm{mO}_{Z}$ ). Then $E=\operatorname{Proj}(\operatorname{gr}(A))([8], 19.4 .2)$. But by our choice of $f$ we have $\operatorname{gr}(A)=$ $\operatorname{gr}(\hat{A}) \cong k[U, V] /\left(U V^{2}\right)$, and hence $E$ consists of exactly two points $P, Q$ which correspond to the ideals $(U)$ and $(V)$ respectively. Put $B=O_{Z, P}$. Since $Z$ is finite we have $B=A\left[x / y, t^{-1}\right]$ where $x, y$ are the images of $X, Y$ in $A$ and $t$ is a suitable element of $A[x / y]$. Hence $B$ is a finitely generated $A$-algebra. Moreover $P$ is a reduced point of $E$, which implies that $\mathfrak{m} B$ is the maximal ideal of $B$. It follows that $B$ is a DVR. Choose now $k$ of characteristic zero: then $B$ is excellent and the proof is complete.

\section{Absolutely flat algebras over excellent rings.}

5.1. Definition. A ring homomorphism $A \rightarrow B$ is said to be:

i) Absolutely flat $(A F)$ if it is reduced, has discrete fibers, and $k(\mathfrak{P})$ is (separable) algebraic over $k(\mathfrak{p})$ whenever $\mathfrak{P}$ lies over $\mathfrak{p}$.

ii) ind-étale if it is an inductive limit of étale homomorphisms. Clearly ind-étale implies $A F$.

5.2. Remark. Since we are dealing with noetherian rings only the above definition of AF agrees with the usual one (i.e. $B$ is flat over $A$ and over $B \otimes_{A} B$ ), as follows by [3], 4.1 and [12], 3.1.

5.3. THEOREM. Let $f: A \rightarrow B$ be an $A F$ ring homomorphism. Then:

i) If $A$ is a P-ring (section 1), then $B$ is a P-ring;

ii) If $A$ is Nagata and J2, the same hold for $B$;

iii) If $A$ is locally Nagata and $U C$, the same hold for $B$;

iv) If $A$ is excellent, $B$ is excellent;

v) If $f$ is faithfully flat the converses of i) and ii) hold;

vi) If $f$ is faithfully flat and $B$ is locally a domain the converses 
of iii) and iv) hold.

Proof. Clearly iv) follows from i), ii), iii); moreover ii) and v) are in $[5](4.8,2.9,4.11)$. To prove i) we may assume $f$ is a local homomorphism of local rings. With the same notations as in 1.3 we have that $\hat{f}$ is formally smooth and hence regular by [1]. Then $v \circ f$ is a $P$ homomorphism, and the conclusion follows by AF, as in [8], proof of 18.3.2. In order to prove iii) and vi) we need the following proposition.

5.4. Proposition. Absolute flatness is stable by finite type base change (i.e. if $A \rightarrow B$ is $A F$ and $A^{\prime}$ is a finite type A-algebra the induced homomorphism $A^{\prime} \rightarrow B^{\prime}=A^{\prime} \otimes_{A} B$ is $\left.A F\right)$.

Proof ${ }^{1)}$. We have only to show that $A^{\prime} \rightarrow B^{\prime}$ has discrete fibers and algebraic residue field extension. We may assume $A^{\prime}=A[X]$, whence $B^{\prime}=B[X]$. Let $\mathfrak{P}^{\prime} \in \operatorname{Spec}\left(B^{\prime}\right)$ and let $\mathfrak{P}, \mathfrak{p}^{\prime}, \mathfrak{p}$ be the contractions of $\mathfrak{P}^{\prime}$ to $B, A^{\prime}, A$ respectively. Tensoring with $k(\mathfrak{p})$ we see that it is sufficient to study the fibers of the induced map $g: k(\mathfrak{p})[X] \rightarrow\left(B \otimes_{A} k(\mathfrak{p})\right)[X]$. By assumption we have $B \otimes_{A} k(p)=K_{1} \times \cdots \times K_{n}$ where the $K_{i}$ 's are algebraic field extensions of $k(\mathfrak{p})$. Thus $g$ is integral and the conclusion follows.

Proof of 5.3. iii). $B$ is locally Nagata by [4]. Then by 5.4 it is sufficient to prove that $B$ is catenarian if $A$ is. Let $\mathfrak{P}_{0} \subsetneq \cdots \sqsubseteq \mathfrak{P}_{n}$ be a saturated chain of prime ideals of $B$ and put $\mathfrak{p}_{i}=f^{-1}\left(\mathfrak{P}_{i}\right)$. The $\mathfrak{p}_{i}$ 's are all distinct by the discreteness of fibers; thus it is enough to show that $\operatorname{dim} A_{\mathfrak{p}_{i}} / \mathfrak{p}_{i-1} A_{\mathfrak{p}_{i}}=1$ for all $i$ 's. This is a consequence of:

5.5. LEMMA. Let $C$ be a UC local domain whose integral closure $C^{\prime}$ is finite. Let $D$ be a local ring and $f: C \rightarrow D$ a local AF homomorphism. Then $\operatorname{dim} D / \mathfrak{P}=\operatorname{dim} C$ for any minimal prime $\mathfrak{P}$ of $D$.

Proof. Since $f$ is AF, $D$ is reduced and its integral closure is $D^{\prime}=C^{\prime} \otimes_{C} D([4], 3.9)$. Then $D^{\prime}=E \times F$, where $E$ is the integral closure of $D / \Re$. By 5.4 the induced map $C^{\prime} \rightarrow D^{\prime}$ is $A F$ and hence the same holds for $C^{\prime} \rightarrow E$. Let $\mathfrak{n}$ be a maximal ideal of $E$ such that $\operatorname{dim} D / \mathfrak{P}=$ $\operatorname{dim} E_{\mathfrak{n}}$, and let $\mathfrak{m}$ be the contraction of $\mathfrak{n}$ to $C^{\prime}$. We have $\operatorname{dim} C_{\mathfrak{m}}^{\prime}=$ $\operatorname{dim} E_{\mathfrak{n}}([4], 3.13)$ and $\operatorname{dim} C=\operatorname{dim} C_{\mathfrak{m}}^{\prime}([8], 5.6 .10)$. The conclusion follows.

1) With the usual definition of AF (see 5.2) this is an immediate consequence of [12], Lemma b. However we prefer to give the present proof, so that everything in this section depends only on Definition 5.1. 
Proof of 5.3. vi). It is sufficient to show that $A$ is catenarian (by 5.4), we may assume $A$ and $B$ are local. The conclusion follows then from 3.3 (see e.g. [4], 3.13 for hypothesis b) of 3.3). This concludes the proof of 5.3 .

5.6. COROLLARY. Let $B$ be a strict henselization of the local ring A. Then we have:

i) $A$ is a P-ring if and only if $B$ is a P-ring;

ii) $A$ is $Q E$ if and only if $B$ is $Q E$;

iii) if $A$ is excellent, $B$ is excellent;

iv) if $B$ is a domain (that is; $A$ is a geometrically unibranche domain, e.g. a normal domain) then $B$ is excellent if and only if $A$ is excellent.

Proof. $B$ is an ind-étale $A$-algebra ([8], 18.8.17).

5.7. Remarks. i) Theorem 5.3 was proved in [5], with the extra assumption: $[k(\mathfrak{M}): k(\mathfrak{m})]$ is finite whenever $\mathfrak{m}$ is the contraction of the maximal ideal $\mathfrak{M}$.

ii) One can prove that $\mathrm{AF}$ homomorphisms preserve Nagata rings (see [12] or [4]).

iii) The converse of 5.3 iii) is false in general ([8], 18.7.7).

iv) Corollary $5.6 \mathrm{i})$, iii) is proved in [16] when the residue field of $A$ is perfect.

\section{BIBLIOGRAPHY}

[1] André, M., Localisation de la lissité formelle, Man. Math., 1974.

[2] Atiyah, M. F. and Macdonald, I. G., Introduction to Commutative Algebra, Addison-Wesley, Reading (Mass.), 1969.

[ 3 ] Ferrand, D., Monomorphismes et morphismes absolument plats, Bull. Soc. Math. France, 100 (1972), 97-128.

[4] Greco, S., Sugli omomorfismi piatti e non ramificati, Le Matematiche (Catania), 24 (1969), 392-415.

[ 5 ] _ - Sugli omomorfismi quasi étale e gli anelli eccellenti, Ann. Mat. Pura ed Appl., 90 (1971), 281-296.

[ 6 ] - and Salmon, P., Topics in m-adic topologies, Erg. der Math. b. 58, Springer Verlag, 1971.

[ 7 ] Grothendieck, A. and Dieudonné, J., Eléments de Géométrie Algébrique I, Grund. der Math. b. 166, Springer Verlag, 1971.

[8] —-, Eléments de Géométrie Algébrique IV, Publ. Math. 20 . ., IHES.

[ 9 ] Matsumura, H., Commutative Algebra, W.A. Benjamin Inc., 1970.

[10] —-, Formal power series rings over polynomial rings I, Number Theory, Algebraic Geometry, and Commutative Algebra in honor of Y. Akizuki, Tokio, 1973, $511-520$. 
[11] Nomura, M., Formal power series rings over polynomial rings II, Number Theory, Algebraic Geometry and Commutative Algebra in honor of Y. Akizuki, Tokio, 1973, 521-528.

[12] Olivier, J. P., Montée des propriétés par morphismes absolument plats, C. R. des Journées d'Algèbre Pure et Appliquée, Montpellier, 1971, 86-109.

[13] Pedrini, C., Incollamenti di ideali primi e gruppi di Picard, Rend. Sem. Mat. Univ. Padova, 48 (1973), 39-66.

[14] Ratliff, L. J. Jr., Catenary rings and the altitude formula, Amer. J. Math., 94 (1972), 458-466.

[15] Seydi, H., Anneaux henséliens et conditions des chaines, Bull. Soc. Math. France, $98(1970), 9-31$.

[16] — Sur la théorie des anneaux de Weierstrass I, Bull. Sc. Math., 96 (1971), $227-235$.

[17] — Sur la théorie des anneaux excellents en caractéristique $p$, I, Bull. Sc. Math., 96 (1972), 193-198.

[18] Valabrega, P., On two-dimensional regular local rings and a lifting problem, Ann. Sc. Norm. Sup. Pisa, 27 (1973), 1-21.

[19] _ - On the excellent property for rings of restricted power series, Boll. UMI, 9 (1974), 486-494.

[20] - On the excellent property for power series rings over polynomial rings, Kyoto J. Math., Vol. 15, No. 2, 1975.

Istituto Matematico del Politecnico di Torino 